\title{
Convolutions in $(\mu, \nu)$-pseudo-almost periodic and $(\mu, \nu)$-pseudo-almost automorphic function spaces and applications to solve integral equations
}

\author{
DAVID BÉKOLLÈ ${ }^{1}$ \\ Khalil EzZINBI ${ }^{2}$ \\ SAMIR FATAJOU ${ }^{3}$ \\ Duplex Elvis Houpa Danga ${ }^{4}$ \\ Fritz Mbounja BÉssÉmè 5 (iD \\ ${ }^{1,4}$ Department of Mathematics, Faculty \\ of Science, University of Ngaoundéré \\ P.O. Box 454, Ngaoundéré, Cameroon. \\ dbekolle@univ-ndere. cm; \\ e_houpa@yahoo.com \\ 2,3 Department of Mathematics, Faculty \\ of Science Semlalia, Cadi Ayyad \\ University, B.P. 2390 Marrakesh, \\ Morocco. \\ ezzinbi@uca.ac.ma; \\ fatajou@yahoo.fr \\ 5 Department of Mines and Geology, \\ School of Geology and Mining \\ Engineering, University of Ngaoundéré \\ P.O. Box 454, Ngaoundéré, Cameroon. \\ mbounjafritz@gmail.com
}

\begin{abstract}
In this paper we give sufficient conditions on $k \in L^{1}(\mathbb{R})$ and the positive measures $\mu, \nu$ such that the doubly-measure pseudo-almost periodic (respectively, doubly-measure pseudoalmost automorphic) function spaces are invariant by the convolution product $\zeta f=k * f$. We provide an appropriate example to illustrate our convolution results. As a consequence, we study under Acquistapace-Terreni conditions and exponential dichotomy, the existence and uniqueness of $(\mu, \nu)$ pseudo-almost periodic (respectively, $(\mu, \nu)$ - pseudo-almost automorphic) solutions to some nonautonomous partial evolution equations in Banach spaces like neutral systems.
\end{abstract}

\section{RESUMEN}

En este artículo damos condiciones suficientes sobre $k \in L^{1}(\mathbb{R})$ y las medidas positivas $\mu, \nu$ tales que los espacios de funciones pseudo-casi periódicas que duplican la medida (respectivamente, pseudo-casi automorfas que duplican la medida) son invariantes por el producto de convolución $\zeta f=k * f$. Entregamos un ejemplo apropiado para ilustrar nuestros resultados de convolución. Como consecuencia, estudiamos bajo condiciones de Acquistapace-Terreni y dicotomía exponencial, la existencia y unicidad de soluciones $(\mu, \nu)$ - pseudo-casi periódicas (respectivamente, $(\mu, \nu)$ - pseudo-casi automorfas) de algunas ecuaciones de evolución parciales no autónomas en espacios de Banach como sistemas neutrales.

Keywords and Phrases: Measure theory, $(\mu, \nu)$-ergodic, $(\mu, \nu)$-pseudo almost periodic and automorphic functions, evolution families, nonautonomous equations, neutral systems.

2020 AMS Mathematics Subject Classification: 34C27, 34K14, 35B15, 35K57, 37A30, 43A60.

\section{(cc) BY-NC}




\section{Introduction}

The existence and uniqueness of pseudo almost periodic and pseudo almost automorphic solutions is one of the most powerful tools in the qualitative theory of differential equations due to applications in mathematical biology, control theory and physical sciences. Recently, Diagana, Ezzinbi and Miraoui [11] applied the abstract measure theory to define the notion of double-weight pseudo almost periodicity (respectively double-weight pseudo almost automorphy) functions, and thus the classical theory of $\mu$-pseudo almost periodic (respectively $\mu$-pseudo almost automorphic) introduced by $[4,5]$, and double-weight pseudo almost periodicity [8] become particular cases of this approach. See the section 2.1 for technical details about this concept of double-weight pseudo almost periodicity (respectively double-weight pseudo almost automorphy) functions. We note that for $f \in P A P(\mathbb{R} \times X, X, \mu, \nu)$ or $f \in P A A(\mathbb{R} \times X, X, \mu, \nu), k \in L^{1}(\mathbb{R}), k * f=k * g+k * \phi$. We have that $k * g$ is almost periodic or almost automorphic function, but $k * \phi$ is not necessarily in $\mathcal{E}(\mathbb{R}, X, \mu, \nu)$. Then, the convolution invariance of the spaces $P A P(\mathbb{R} \times X, X, \mu, \nu)$ (resp. $P A A(\mathbb{R} \times X, X, \mu, \nu))$ is equivalent to the convolution invariance of $\mathcal{E}(\mathbb{R}, X, \mu, \nu)$.

During the last decade, many research results about pseudo almost periodic and pseudo almost atomorphic was produce see $[4,5,7,9,10]$. Inspired by the work of Ezzinbi et al. [11] who studied the translation invariance of $P A A(\mathbb{R} \times X, X, \mu, \nu)$ (resp. $P A P(\mathbb{R} \times X, X, \mu, \nu))$ functions and the recent work of Mbounja et al. [15] who gave some several hypotheses for convolution invariance of $P A P(\mathbb{R} \times X, X, \mu)$ and $P A A(\mathbb{R} \times X, X, \mu)$, in this work we established new sufficient conditions on $\mu, \nu \in \mathcal{M}$ and $k \in L^{1}(\mathbb{R})$ ensuring that, the space $P A P(\mathbb{R}, X, \mu, \nu)$ of $(\mu, \nu)$-pseudo almost periodic functions and the space $P A A(\mathbb{R}, X, \mu, \nu)$ of $(\mu, \nu)$-pseudo almost automorphic functions are invariant by the convolution product $\zeta f=k * f$. Our obtained conditions are more general than [15] and helped to show that the integral solution of some differential equations is a $(\mu, \nu)$-pseudo almost periodic (respectively $(\mu, \nu)$-pseudo almost automorphic) solutions. To illustrate our investigation, we show the existence and uniqueness of $(\mu, \nu)$-pseudo almost periodic (respectively $(\mu, \nu)$-pseudo almost automorphic) solutions of the following nonautomous differential equations,

$$
\frac{d}{d t} u(t)=A(t) u(t)+F(t, u(t)), \quad t \in \mathbb{R},
$$

and

$$
\frac{d}{d t}(u(t)-G(t, u(t))=A(t)(u(t)-G(t, u(t))+F(t, u(t)), \quad t \in \mathbb{R},
$$

where $A(t): D(A(t)) \subset X \longmapsto X$ for $t \in \mathbb{R}$ is a family of closed linear operators on a Banach space $X$, satisfying the well-known Acquitaspace-Terreni conditions developed in [1,2], and $F, G: \mathbb{R} \times X \longmapsto X$ are jointly continuous functions satisfying some additional conditions. The study of equation (1.1) in an non-autonomous case is new even in the case of one measure, $\mu=\nu$. Also, equation (1.2) is treated here.

The rest of this work is organized as follows. In section 2, we recall some basic results which will be used throughout this work. In section 3, we state and prove main results about the convolution 
invariance. In section 4 we study the existence and uniqueness of $(\mu, \nu)$-pseudo almost periodic (respectively $(\mu, \nu)$-pseudo almost automorphic) solutions to both equation (1.1) and equation (1.2) which illustrate our new results.

\section{Preliminaries}

\subsection{Notation and terminology}

Let $(X,\|\cdot\|)$ a Banach space and let $B C(\mathbb{R}, X)$ be the space of bounded continuous functions $f: \mathbb{R} \longrightarrow X$. The space $B C(\mathbb{R}, X)$, equipped with the supremum norm $\|f\|_{\infty}=\sup _{t \in \mathbb{R}}\|f(t)\|$, is a Banach space.

We denote by $\mathcal{B}$ the Lebesgue $\sigma$-field of $\mathbb{R}$ and by $\mathcal{M}$ the space of all positive measures $\vartheta$ on $\mathcal{B}$ satisfying $\vartheta(\mathbb{R})=+\infty$ and $\vartheta([a, b])<\infty$, for all $a, b \in \mathbb{R}(a \leq b)$.

Definition $2.1([6])$. A continuous function $f: \mathbb{R} \rightarrow X$ is said to be almost periodic if for every $\varepsilon>0$ there exists a positive number $l_{\varepsilon}$ such that every interval of length $l_{\varepsilon}$ contains a number $\tau$ such that:

$$
\|f(t+\tau)-f(t)\|<\varepsilon, \quad \forall t \in \mathbb{R} .
$$

Let $A P(\mathbb{R}, X)$ denote the collection of almost periodic functions from $\mathbb{R}$ to $X$. We recall that $\left(A P(\mathbb{R}, X),\|\cdot\|_{\infty}\right)$ is a Banach space.

Definition $2.2([11])$. Let $\mu, \nu \in \mathcal{M}$. A bounded continuous function $f: \mathbb{R} \rightarrow X$ is said to be $(\mu, \nu)$-ergodic if

$$
\lim _{r \rightarrow+\infty} \frac{1}{\nu([-r, r])} \int_{-r}^{r}\|f(t)\| d \mu(t)=0 .
$$

We denote the space of all such functions by $\mathcal{E}(\mathbb{R}, X, \mu, \nu)$.

The space $\left(\mathcal{E}(\mathbb{R}, X, \mu, \nu),\|\cdot\|_{\infty}\right)$ is a Banach space for the supremum norm.

Definition $2.3([11])$. Let $\mu, \nu \in \mathcal{M}$. A continuous function $f: \mathbb{R} \rightarrow X$ is said to be $(\mu, \nu)$-pseudo almost periodic if $f$ admits the following decomposition:

$$
f=g+\phi
$$

where $g \in A P(\mathbb{R}, X)$ and $\phi \in \mathcal{E}(\mathbb{R}, X, \mu, \nu)$.

We denote the space of all such functions by $P A P(\mathbb{R}, X, \mu, \nu)$. 
We have $A P(\mathbb{R}, X) \subset P A P(\mathbb{R}, X, \mu, \nu) \subset B C(\mathbb{R}, X)$.

Let $(Y,\|\cdot\|)$ a Banach space and let $B C(\mathbb{R} \times Y, X)$ be the space of jointly bounded continuous functions $f: \mathbb{R} \times Y \longrightarrow X$. The space $B C(\mathbb{R} \times Y, X)$ equipped with the supremum norm $\|f\|_{\infty}=\sup _{t \in \mathbb{R}, x \in Y}\|f(t, x)\|$ is a Banach space.

Definition 2.4 ([12]). A jointly continuous function $f: \mathbb{R} \times Y \rightarrow X$ is said to be almost periodic in $t$ uniformly with respect to $x \in Y$, if for every $\varepsilon>0$, and any compact subset $K$ of $Y$, there exists a positive number $l_{K}(\varepsilon)$ such that every interval of length $l_{K}(\varepsilon)$ contains a number $\tau$ such that:

$$
\|f(t+\tau, x)-f(t, x)\|<\varepsilon, \quad \forall(t, x) \in \mathbb{R} \times K .
$$

We denote the space of such functions by $A P U(\mathbb{R} \times Y, X)$.

Definition 2.5 ([11]). Let $\mu, \nu \in \mathcal{M}$. A continuous function $f: \mathbb{R} \times Y \rightarrow X$ is said to be $(\mu, \nu)$-ergodic in $t$ uniformly with respect to $x \in Y$, if the following two conditions are true:

(i) $f$ is uniformly continuous on each compact set $K$ in $Y$ with respect to the second variable $x$.

(ii) $\forall x \in Y, f(., x) \in \mathcal{E}(\mathbb{R}, X, \mu, \nu)$.

The space of such functions is denoted by $\mathcal{E} U(\mathbb{R} \times Y, X, \mu, \nu)$.

Definition 2.6 ([11]). Let $\mu, \nu \in \mathcal{M}$. A continuous function $f: \mathbb{R} \times Y \rightarrow X$ is said to be $(\mu, \nu)$-pseudo almost periodic in $t$ uniformly for $x \in Y$, if $f$ admits the following decomposition:

$$
f=g+\phi
$$

where $g \in A P U(\mathbb{R} \times Y, X)$ and $\phi \in \mathcal{E} U(\mathbb{R} \times Y, X, \mu, \nu)$.

The collection of such functions is denoted by $P A P U(\mathbb{R} \times Y, X, \mu, \nu)$.

We have $A P U(\mathbb{R} \times Y, X) \subset P A P U(\mathbb{R} \times Y, X, \mu, \nu) \subset B C(\mathbb{R} \times Y, X, \mu, \nu)$.

Definition 2.7 ([16]). A continuous function $f: \mathbb{R} \rightarrow X$ is said to be almost automorphic if for every sequence of real numbers $\left(s_{n}^{\prime}\right)_{n \in \mathbb{N}}$, there exists a subsequence $\left(s_{n}\right)_{n \in \mathbb{N}} \subset\left(s_{n}^{\prime}\right)_{n \in \mathbb{N}}$ such that:

$$
\lim _{n, m \rightarrow \infty} f\left(t+s_{n}-s_{m}\right)=f(t), \quad \text { for } \quad \text { each } \quad t \in \mathbb{R}
$$

Equivalently,

$$
g(t)=\lim _{n \rightarrow \infty} f\left(t+s_{n}\right) \quad \text { exists } \quad \forall t \in \mathbb{R} \quad \text { and } \quad f(t)=\lim _{n \rightarrow \infty} g\left(t-s_{n}\right) \quad \forall t \in \mathbb{R} .
$$

We denote the space of such functions by $A A(\mathbb{R}, X)$. 
We recall that $\left(A A(\mathbb{R}, X),\|\cdot\|_{\infty}\right)$ is a Banach space.

Definition 2.8 ([11]). Let $\mu, \nu \in \mathcal{M}$. A continuous function $f: \mathbb{R} \rightarrow X$ is said to be $(\mu, \nu)$-pseudo almost automorphic if $f$ admits the following decomposition:

$$
f=g+\phi
$$

where $g \in A A(\mathbb{R}, X)$ and $\phi \in \mathcal{E}(\mathbb{R}, X, \mu, \nu)$.

We denote the space of all such functions by $P A A(\mathbb{R}, X, \mu, \nu)$.

We have $A A(\mathbb{R}, X) \subset P A A(\mathbb{R}, X, \mu, \nu) \subset B C(\mathbb{R}, X)$.

Definition 2.9 ([16]). A continuous function $f: \mathbb{R} \times Y \rightarrow X$ is said to be almost automorphic in $t$ uniformly for $x \in Y$, if the following conditions hold:

(i) $f$ is uniformly continuous on each compact set $K$ in $Y$ with respect to the second variable $x$, namely, for each compact set $K$ in $Y$, for all $\varepsilon>0$, there exists $\delta>0$ such that for all $x_{1}, x_{2} \in K$, one has:

$$
\left\|x_{1}-x_{2}\right\| \leq \delta \Rightarrow \sup _{t \in \mathbb{R}}\left\|f\left(t, x_{1}\right)-f\left(t, x_{2}\right)\right\| \leq \varepsilon
$$

(ii) for all $x \in Y, f(., x) \in A A(\mathbb{R}, X)$.

Denote by $A A U(\mathbb{R} \times Y, X)$ the set of all such functions.

Definition 2.10 ([11]). Let $\mu, \nu \in \mathcal{M}$. A continuous function $f: \mathbb{R} \times Y \rightarrow X$ is said to be $(\mu, \nu)$-pseudo almost periodic in $t$ uniformly for $x \in Y$, if $f$ admits the following decomposition:

$$
f=g+\phi
$$

where $g \in A A U(\mathbb{R} \times Y, X)$ and $\phi \in \mathcal{E} U(\mathbb{R} \times Y, X, \mu, \nu)$.

The collection of such functions is denoted by $P A A U(\mathbb{R} \times Y, X, \mu, \nu)$

We have $A A U(\mathbb{R} \times Y, X) \subset P A A U(\mathbb{R} \times Y, X, \mu, \nu) \subset B C(\mathbb{R} \times Y, X, \mu, \nu)$

\subsection{Some useful results on the space functions}

For $\mu \in \mathcal{M}$ and $\tau \in \mathbb{R}$, we denote by $\mu_{\tau}$ the positive measure on $(\mathbb{R}, \mathcal{B})$ defined by:

$$
\mu_{\tau}(A)=\mu(\{a+\tau: a \in A\}), \quad \forall A \in \mathcal{B} .
$$


Now we introduce the following hypotheses on $\mu, \nu \in \mathcal{M}$.

$\left(\mathbf{H}_{0}\right)$ : For all $\tau \in \mathbb{R}$, there exists $\delta>0$ and a bounded interval $I$ such that

$$
\mu_{\tau}(A) \leq \delta \mu(A), \quad \nu_{\tau}(A) \leq \delta \nu(A), \quad \forall A \in \mathcal{B} \quad \text { satisfied } \quad A \cap I=\emptyset .
$$

$\left(\mathbf{H}_{1}\right)$ :

$$
\limsup _{r \rightarrow \infty} \frac{\mu([-r, r])}{\nu([-r, r])}<\infty
$$

\section{Remark 2.11.}

i) Without assumptions on $\mu$ and $\nu$, like $\left(\boldsymbol{H}_{0}\right)$, the decomposition (2.1) (resp. (2.2)) of the $(\mu, \nu)$ pseudo almost periodic and automorphic functions is not unique, (see [11]).

ii) The spaces $\mathcal{E}(\mathbb{R}, X, \mu, \nu), \mathcal{E}(\mathbb{R} \times Y, X, \mu, \nu), P A P(\mathbb{R}, X, \mu, \nu), P A P(\mathbb{R} \times Y, X, \mu, \nu), P A A(\mathbb{R}, X, \mu, \nu)$, and $P A A(\mathbb{R} \times Y, X, \mu, \nu)$ coincides when $\mu=\nu$, with the spaces $\mathcal{E}(\mathbb{R}, X, \mu), \mathcal{E}(\mathbb{R} \times Y, X, \mu)$, $P A P(\mathbb{R}, X, \mu), P A P(\mathbb{R} \times Y, X, \mu), P A A(\mathbb{R}, X, \mu)$, and $P A A(\mathbb{R} \times Y, X, \mu)$.

We recall the following six theorems proved in [11].

Theorem $2.12([11])$. Consider that $\mu, \nu \in \mathcal{M}$ and $k \in L^{1}(\mathbb{R})$ and $f \in P A P(\mathbb{R}, X, \mu, \nu)$ (respectively $f \in P A A(\mathbb{R}, X, \mu, \nu)$. If $\left(\boldsymbol{H}_{0}\right)$ is valid then $P A P(\mathbb{R}, X, \mu, \nu)$ (respectively $P A A(\mathbb{R}, X, \mu, \nu)$ ) is translation invariant. Moreover,

$$
\{g(t): t \in \mathbb{R}\} \subset \overline{\{f(t): t \in \mathbb{R}\}} \text {, (the closure of the range of } f \text { ). }
$$

Theorem $2.13([11]) . \quad$ If $\left(\boldsymbol{H}_{0}\right)$ is valid, then the decomposition $(2.1)$ (resp. (2.2)) of PAP $(\mathbb{R}, X, \mu, \nu)$ and $P A A(\mathbb{R}, X, \mu)$ is unique.

Theorem $2.14([11])$. If $\left(\boldsymbol{H}_{1}\right)$ holds, then $\left(\mathcal{E}(\mathbb{R}, X, \mu, \nu),\|\cdot\|_{\infty}\right)$ is a Banach space with respect to the sup norm.

Theorem 2.15 ([11]). Let $\mu, \nu \in \mathcal{M}$ satisfy $\left(\boldsymbol{H}_{1}\right)$. If $\left(\boldsymbol{H}_{0}\right)$ holds, then $P A P(\mathbb{R}, X, \mu, \nu)$ and $P A A(\mathbb{R}, X, \mu, \nu)$ are Banach spaces with respect to the sup norm.

Theorem 2.16 ([11]). Let $\mu, \nu \in \mathcal{M}, F \in P A P U(\mathbb{R} \times Y, X, \mu, \nu)$ and $h \in P A P(\mathbb{R}, X, \mu, \nu)$. Assume that $\left(\boldsymbol{H}_{1}\right)$ and the following hypothesis holds:

For all bounded subsets $B$ of $X, F$ is bounded on $\mathbb{R} \times B$.

Then $t \longmapsto F(t, h(t)) \in P A P(\mathbb{R}, X, \mu, \nu)$.

Theorem 2.17 ([11]). Let $\mu, \nu \in \mathcal{M}, F \in P A A U(\mathbb{R} \times Y, X, \mu, \nu)$ and $h \in P A A(\mathbb{R}, X, \mu, \nu)$. Assume that for all bounded subsets $B$ of $X, F$ is bounded on $\mathbb{R} \times B$. Then $t \longmapsto F(t, h(t)) \in$ $P A A(\mathbb{R}, X, \mu, \nu)$. 


\subsection{Measure theory results}

Let $\mu, \nu \in \mathcal{M}$; if $f: \mathbb{R} \longrightarrow X$ is a bounded continuous function, we define the following doublyweight mean, if the limit exists, by:

$$
\mathbf{M}(f, \mu, \nu):=\lim _{r \rightarrow+\infty} \frac{1}{\nu([-r, r])} \int_{-r}^{r}\|f(t)\|_{X} d \mu(t) .
$$

Definition $2.18([17])$. Let $(E, \mathcal{B})$ be a Borel space. If $\mu$ and $\nu$ are two measures defined on $(E, \mathcal{B})$, we say that:

(i) $\mu$ and $\nu$ are mutually singular, if there are disjoint sets $A$ and $B$ in $\mathcal{B}$ such that $E=A \cup B$ and

$$
\nu(A)=\mu(B)=0
$$

(ii) $\nu$ is absolutely continuous with respect to $\mu$, if for each $A \in \mathcal{B}$,

$$
(\mu(A)=0) \Longrightarrow(\nu(A)=0) .
$$

We recall the following theorems of measure theory.

Theorem 2.19 (Radon-Nikodym [17]). Let $(E, \mathcal{B}, \mu)$ be a $\sigma$-finite measure space, and let $\nu$ be a measure defined on $\mathcal{B}$ which is absolutely continuous with respect to $\mu$. Then there is a unique nonnegative measurable function $f$ such that for each set $B$ in $\mathcal{B}$ we have:

$$
\nu(B)=\int_{B} f d \mu \text {. }
$$

The function $f$ is called the Radon-Nikodym derivative of $\nu$ with respect of $\mu$.

\section{Example 2.20.}

Let $\rho$ be a nonnegative $\mathcal{B}$-measurable function. Denote by $\mu$ the positive measure defined by:

$$
\mu(A)=\int_{A} \rho(t) d t, \quad \text { for } \quad A \in \mathcal{B}
$$

where $d t$ is the Lebesgue measure on $\mathbb{R}$. The function $\rho$ is the Radon-Nikodym derivative of $\mu$ with respect to the Lebesgue measure $d t$ on $\mathbb{R}$, i.e. $d \mu(t)=\rho(t) d t$. In this case, $\mu \in \mathcal{M}$ if and only if its Radon-Nikodym derivative $\rho$ is locally Lebesgue integrable on $\mathbb{R}$ and it satisfies

$$
\int_{-\infty}^{+\infty} \rho(t) d t=+\infty
$$

Theorem 2.21 (Lebesgue-Radon-Nikodym [17]). $\quad$ Let $(X, \mathcal{B}, \vartheta)$ be a $\sigma$-finite measure space, and $\mu$ a $\sigma$-finite measure defined on $\mathcal{B}$. Then, we can find a measure $\mu_{0}$, singular with respect to $\vartheta$, and a measure $\mu_{1}$, absolutely continuous with respect to $\vartheta$, such that $\mu=\mu_{0}+\mu_{1}$. The measures $\mu_{0}$ and $\mu_{1}$ are unique. 
In this section, by using the previous theorem, we consider that for a given $\mu \in \mathcal{M}, \mu=\mu_{0}+\mu_{1}$ where $\mu_{0}$ is the $\mu$-measure component which is absolutely continuous with respect to the Lebesgue measure and its Radon-Nikodym derivative is $\rho$, that is $d \mu_{0}(t)=\rho(t) d t$ and $\mu_{1}$ is the $\mu$-measure component such that $\mu_{1}$ is singular to Lebesgue measure.

We give new general hypotheses on $\mu, \nu \in \mathcal{M}$ and $k \in L^{1}(\mathbb{R})$ such that:

$$
(\zeta f)(t)=\int_{-\infty}^{+\infty} k(t-s) f(s) d s, \quad \forall k \in L^{1}(\mathbb{R})
$$

maps $\mathcal{E}(\mathbb{R}, X, \mu, \nu)$ into itself.

In particular, our hypotheses on $\mu, \nu \in \mathcal{M}$ and $k \in L^{1}(\mathbb{R})$ will imply that for every $f \in \mathcal{E}(\mathbb{R}, X, \mu, \nu)$, the $(\mu, \nu)$-mean,

$$
\mathbf{M}(\zeta f, \mu, \nu):=\lim _{r \rightarrow+\infty} \frac{1}{\nu([-r, r])} \int_{-r}^{r}\left\|\int_{-\infty}^{+\infty} k(t-s) f(s) d s\right\|_{X} d \mu(t)
$$

exists.

\section{Main results of convolution and translation invariance}

\subsection{Convolution invariance on $\mathcal{E}(\mathbb{R}, X, \mu, \nu)$}

Theorem 3.1. Let $k \in L^{1}(\mathbb{R})$ and $\nu \in \mathcal{M}$. Consider that $\mu \in \mathcal{M}$, with Radon-Nikodym derivative $\rho$ with respect to $d t$ and $\zeta$ is defined in (2.3). Assume that $\rho, \mu, \nu$ and $k$ satisfy the following requirements:

$$
\begin{gathered}
\left\{\begin{array}{l}
\sup _{|s| \leq r, r \in \mathbb{R}_{+}} \frac{1}{\rho(s)} \int_{s}^{r}|k(t-s)| d \mu(t)<\infty, \\
\sup _{|s| \leq r, r \in \mathbb{R}_{+}} \frac{1}{\rho(s)} \int_{-r}^{s}|k(t-s)| d \mu(t)<\infty,
\end{array}\right. \\
\left\{\begin{array}{l}
\lim _{r \rightarrow+\infty} \frac{1}{\nu([-r, r])} \int_{-\infty}^{-r}\left(\int_{-r}^{r}|k(t-s)| d \mu(t)\right) d s=0, \\
\lim _{r \rightarrow+\infty} \frac{1}{\nu([-r, r])} \int_{r}^{+\infty}\left(\int_{-r}^{r}|k(t-s)| d \mu(t)\right) d s=0 .
\end{array}\right.
\end{gathered}
$$

If $f \in \mathcal{E}(\mathbb{R}, X, \mu, \nu)$, then $\zeta f \in \mathcal{E}(\mathbb{R}, X, \mu, \nu)$.

Proof. We adapt the proof in [15], Theorem 3.5. By the properties of convolution we have that $f \in B C(\mathbb{R}, X)$ implies that $k * f \in B C(\mathbb{R}, X), \forall k \in L^{1}(\mathbb{R})$. Then, in order to get that $k * f \in \mathcal{E}(\mathbb{R}, X, \mu, \nu)$ we must prove that $\mathbf{M}(\zeta f, \mu, \nu)=0$.

We consider $\mu \in \mathcal{M}$ and $\rho$ its Radon-Nikodym derivative, $\nu \in \mathcal{M}$. In the first stage, we assume that $k(t)=0$ on $\mathbb{R}_{-}^{*}$. From $\nu(\mathbb{R})=+\infty$, we deduce the existence of $r_{0} \geq 0$ such that $\nu([-r, r])>$ $0, \forall r \geq r_{0}$. Then by applying the Fubini's Theorem, we deduce that for $f \in B C(\mathbb{R}, X), \forall r \geq r_{0}$. 
We notice that

$$
\begin{aligned}
& \mathbf{M}(\zeta f, \mu, \nu)=\lim _{r \rightarrow+\infty} \frac{1}{\nu([-r, r])} \int_{-r}^{r}\|k * f\|_{X} d \mu(t) \\
& \leq \lim _{r \rightarrow+\infty} \frac{1}{\nu([-r, r])} \int_{-r}^{r} \int_{-\infty}^{t}\|f(s)\|_{X}|k(t-s)| d s d \mu(t) \\
& =\lim _{r \rightarrow+\infty} \frac{1}{\nu([-r, r])} \int_{-r}^{r}\left(\int_{-\infty}^{-r}\|f(s)\|_{X}|k(t-s)| d s\right) d \mu(t) \\
& +\lim _{r \rightarrow+\infty} \frac{1}{\nu([-r, r])} \int_{-r}^{r}\left(\int_{-r}^{t}\|f(s)\|_{X}|k(t-s)| d s\right) d \mu(t) \\
& \leq\|f\|_{\infty} \lim _{r \rightarrow+\infty} \frac{1}{\nu([-r, r])} \int_{-r}^{r}\left(\int_{-\infty}^{-r}|k(t-s)| d s\right) d \mu(t) \\
& +\lim _{r \rightarrow+\infty} \frac{1}{\nu([-r, r])} \int_{-r}^{r}\|f(s)\|_{X}\left(\int_{s}^{r}|k(t-s)| d \mu(t)\right) d s \\
& \leq\|f\|_{\infty} \lim _{r \rightarrow+\infty} \frac{1}{\nu([-r, r])} \int_{-\infty}^{-r}\left(\int_{-r}^{r}|k(t-s)| d \mu(t)\right) d s \\
& +\lim _{r \rightarrow+\infty} \frac{1}{\nu([-r, r])} \int_{-r}^{r}\|f(s)\|_{X}\left[\frac{1}{\rho(s)} \int_{s}^{r}|k(t-s)| d \mu(t)\right] \rho(s) d s \\
& \leq \sup _{|s| \leq r, r \in \mathbb{R}_{+}} \frac{1}{\rho(s)} \int_{s}^{r}|k(t-s)| d \mu(t) \lim _{r \rightarrow+\infty} \frac{1}{\nu([-r, r])} \int_{-r}^{r}\|f(s)\|_{X} \rho(s) d s \\
& +\|f\|_{\infty} \lim _{r \rightarrow+\infty} \frac{1}{\nu([-r, r])} \int_{-\infty}^{-r}\left(\int_{-r}^{r}|k(t-s)| d \mu(t)\right) d s \\
& \leq \sup _{|s| \leq r, r \in \mathbb{R}_{+}} \frac{1}{\rho(s)} \int_{s}^{r}|k(t-s)| d \mu(t) \lim _{r \rightarrow+\infty} \frac{1}{\nu([-r, r])} \int_{-r}^{r}\|f(s)\|_{X} d \mu(s) \\
& +\|f\|_{\infty} \lim _{r \rightarrow+\infty} \frac{1}{\nu([-r, r])} \int_{-\infty}^{-r}\left(\int_{-r}^{r}|k(t-s)| d \mu(t)\right) d s .
\end{aligned}
$$

Using assumptions (3.1.1), (3.2.1) and the fact that $f \in \mathcal{E}(\mathbb{R}, X, \mu, \nu)$, we have proved that $\mathbf{M}(\zeta f, \mu, \nu)=0+0=0$. This settles the first stage for every $k \in L^{1}(\mathbb{R})$ such that $k(t)=0$ on $\mathbb{R}_{-}^{*}$. Now, in the second stage, proceeding similarly like in the first stage, we assume that $k(t)=0$ on $\mathbb{R}_{+}^{*}$ we obtain:

$$
\begin{aligned}
\mathbf{M}(\zeta f, \mu, \nu) & =\lim _{r \rightarrow+\infty} \frac{1}{\nu([-r, r])} \int_{-r}^{r}\|k * f\|_{X} d \mu(t) \\
& \leq\|f\|_{\infty} \lim _{r \rightarrow+\infty} \frac{1}{\nu([-r, r])} \int_{r}^{+\infty}\left(\int_{-r}^{r}|k(t-s)| d \mu(t)\right) d s \\
& +\sup _{|s| \leq r, r \in \mathbb{R}_{+}} \frac{1}{\rho(s)} \int_{-r}^{s}|k(t-s)| d \mu(t) \lim _{r \rightarrow+\infty} \frac{1}{\nu([-r, r])} \int_{-r}^{r}\|f(s)\|_{X} d \mu(s) .
\end{aligned}
$$

Then, using the fact that $f \in \mathcal{E}(\mathbb{R}, X, \mu, \nu)$ and hypotheses (3.1.2), (3.2.2), we have that $\mathbf{M}(\zeta f, \mu, \nu)=0$.

In the general case of $k$, we deduce the result using the fact that $k(t)=k \chi_{t \geq 0}(t)+k \chi_{t<0}(t)$.

Theorem 3.2. Assume that $\mu, \nu \in \mathcal{M}$ and $\left(\boldsymbol{H}_{1}\right)$ holds. Then the condition (3.2.1) (resp. (3.2.2)) 
is valid for every $k \in L^{1}(\mathbb{R})$ if and only if the following condition (3.3.1) (resp. (3.3.2)) is true:

$$
\begin{cases}\lim _{r \rightarrow+\infty} \frac{\mu([-r, \sigma-r])}{\nu([-r, r])}=0, & \forall \sigma>0 \\ \lim _{r \rightarrow+\infty} \frac{\mu([\sigma+r, r])}{\nu([-r, r])}=0, & \forall \sigma<0\end{cases}
$$

Proof. We first prove that $(3.3 .1) \Longrightarrow(3.2 .1)$, for every $k \in L^{1}(\mathbb{R})$.

In the first stage, we assume that $k(t)=0$ on $\mathbb{R}_{-}^{*}$. Let $\sigma=t-s>0$ fixed. From $\nu(\mathbb{R})=+\infty$, we deduce the existence of $r_{0} \geq 0$ such that $\nu([-r, r])>0, \quad \forall r \geq r_{0}$. In the sequel, for all $r \geq r_{0}$, we shall assume that

$$
B:=\frac{1}{\nu([-r, r])} \int_{-r}^{r}\left(\int_{-\infty}^{-r}|k(t-s)| d s\right) d \mu(t) .
$$

Then, by applying the Fubini's Theorem we deduce that:

$$
\begin{aligned}
B & =\frac{1}{\nu([-r, r])} \int_{-r}^{r}\left(\int_{t+r}^{+\infty}|k(\sigma)| d \sigma\right) d \mu(t) \\
& =\int_{0}^{+\infty}\left(\frac{\int_{-r}^{\min (\sigma-r, r)} d \mu(t)}{\nu([-r, r])}\right)|k(\sigma)| d \sigma \\
& =\int_{0}^{+\infty}\left(\frac{\mu([-r, \min (\sigma-r, r)])}{\nu([-r, r])}\right)|k(\sigma)| d \sigma .
\end{aligned}
$$

By using assumption (3.3.1), we have:

$$
\lim _{r \rightarrow+\infty} \frac{\mu([-r, \min (\sigma-r, r)])}{\nu([-r, r])}=0, \quad \forall \sigma>0 .
$$

Since $\mu([-r, \min (\sigma-r, r)]) \leq \mu([-r, r])$ and the fact that $\left(\mathbf{H}_{1}\right)$ holds, there exists $\beta>0$ such that:

$$
0 \leq\left(\frac{\mu([-r, \min (\sigma-r, r)])}{\nu([-r, r])}\right)|k(\sigma)| \leq \beta|k(\sigma)|,
$$

where $k \in L^{1}(\mathbb{R}), \forall \sigma>0$. Then, by the Lebesgue dominated convergence Theorem, we obtain:

$$
\lim _{r \rightarrow+\infty} \int_{0}^{+\infty}\left(\frac{\mu([-r, \min (\sigma-r, r)])}{\nu([-r, r])}\right)|k(\sigma)| d \sigma=0
$$

This concludes this stage of (3.2.1).

Now, in the second stage, proceeding similarly like the first stage, we assume that $k(t)=0$ on $\mathbb{R}_{+}^{*}$. Let $\sigma=t-s<0$ fixed. From $\nu(\mathbb{R})=+\infty$, we deduce the existence of $r_{0} \geq 0$ such that $\nu([-r, r])>0, \nu([-r, r])>0, \quad \forall r \geq r_{0}$. We set:

$$
A:=\frac{1}{\nu([-r, r])} \int_{-r}^{r}\left(\int_{r}^{+\infty}|k(t-s)| d s\right) d \mu(t)
$$


Then, by applying the Fubini's Theorem, we deduce that:

$$
\begin{aligned}
A & =\frac{1}{\nu([-r, r])} \int_{-r}^{r}\left(\int_{-\infty}^{t-r}|k(\sigma)| d \sigma\right) d \mu(t) \\
& =\int_{-\infty}^{0}\left(\frac{\int_{\max (\sigma+r, r)}^{r} d \mu(t)}{\nu([-r, r])}\right)|k(\sigma)| d \sigma \\
& =\int_{-\infty}^{0}\left(\frac{\mu([\max (\sigma+r, r), r])}{\nu([-r, r])}\right)|k(\sigma)| d \sigma .
\end{aligned}
$$

Like in the first part, we use assumptions (3.3.2), and the Lebesgue dominated convergence Theorem. This concludes this second stage of (3.2.2).

Let us prove $(3.2 .1) \Longrightarrow(3.3 .1)$.

Let $\sigma=t-s$, by (3.2.1) and Fubini's Theorem we have that:

$$
\begin{aligned}
0 & =\lim _{r \rightarrow+\infty} \frac{1}{\nu([-r, r])} \int_{-r}^{r}\left(\int_{-\infty}^{-r}|k(t-s)| d s\right) d \mu(t) \\
& =\lim _{r \rightarrow+\infty} \int_{0}^{+\infty}\left(\frac{\mu([-r, \min (\sigma-r, r)])}{\nu([-r, r])}\right)|k(\sigma)| d \sigma .
\end{aligned}
$$

Let $\tau>0$ such that $\sigma \in[\tau, \tau+1]$ and $r>\frac{\tau}{2}$. We have also $[-r, \tau-r] \subseteq[-r, \sigma-r]$ and $[-r, \tau-r] \subseteq[-r, r]$, that implies $\mu([-r, \tau-r]) \leq \min \{\mu([-r, \sigma-r]), \mu([-r, r])\}$, i.e.

$$
\frac{\mu([-r, \tau-r])}{\nu([-r, r])} \leq \frac{\mu([-r, \min (\sigma-r, r)])}{\nu([-r, r])} .
$$

Let $k(\sigma)=\chi_{[\tau, \tau+1]}(\sigma)$. We have that:

$$
\begin{aligned}
0 & \leq \frac{\mu([-r, \tau-r])}{\nu([-r, r])} \int_{\tau}^{\tau+1} d \sigma \\
& \leq \int_{\tau}^{\tau+1} \frac{\mu([-r, \min (\sigma-r, r)])}{\nu([-r, r])} d \sigma \\
& =\lim _{r \rightarrow+\infty} \frac{1}{\nu([-r, r])} \int_{-r}^{r}\left(\int_{-\infty}^{-r}|k(t-s)| d s\right) d \mu(t) .
\end{aligned}
$$

Then by (3.2.1):

$$
\limsup _{r \rightarrow+\infty} \frac{\mu([-r, \tau-r])}{\nu([-r, r])} \int_{\tau}^{\tau+1} d \sigma \leq \lim _{r \rightarrow+\infty} \int_{\tau}^{\tau+1} \frac{\mu([-r, \min (\sigma-r, r)])}{\nu([-r, r])} d \sigma=0
$$

then:

$$
\lim _{r \rightarrow+\infty} \frac{\mu([-r, \tau-r])}{\nu([-r, r])}=0 .
$$

So (3.3.1) is verified. In the second stage (3.3.2), we do the same proof as above. 
Remark 3.3. Hypothesis $\left(\boldsymbol{H}_{1}\right)$ was used only in the proof of the implication $(3.3 .1) \Longrightarrow(3.2 .1)$.

Corollary 3.4. Let $\mu, \nu \in \mathcal{M}$ be such that the nonnegative $\mathcal{B}$-measurable function $\rho$ be the RadonNikodym derivative of $\mu$. Assume that for all $k \in L^{1}(\mathbb{R})$ the requirements (3.1) and (3.2) are satisfied. Then $\mathcal{E}(\mathbb{R}, X, \mu, \nu)$ is convolution invariant.

Corollary 3.5. Consider that $\mu, \nu \in \mathcal{M}$, such that the nonnegative $\mathcal{B}$-measurable function $\rho$ be the Radon-Nikodym derivative of $\mu$. Assume that $\left(\boldsymbol{H}_{1}\right)$ holds and the requirements (3.1) and (3.3) are satisfied. Then $\mathcal{E}(\mathbb{R}, X, \mu, \nu)$ is convolution invariant.

\section{Example 3.6.}

We check that Theorem 3.1 and Corollary 3.5 hold.

Let

$$
k(t)=\left\{\begin{array}{l}
\frac{1}{10} e^{-2 t}, \text { for } t \in[0,+\infty[ \\
0, \text { for } t \in]-\infty, 0[
\end{array}\right.
$$

We take $d \mu_{k, \eta}(t)=e^{\sigma t} d t+\eta \sum_{n=-\infty}^{\infty} e^{\sigma n} \delta_{n}$, where $0 \leq \sigma<2, \quad \eta>0$ and $\delta_{n}$ denotes the Dirac measure at the integer $n\left(\sum_{n=-\infty}^{\infty} e^{\sigma n} \delta_{n}\right.$ is a 'generalized Dirac comb', it is called a Dirac comb when $\sigma=0)$. Then $\mu_{\sigma, \eta} \in \mathcal{M}$ and its Radon-Nikodym derivative is $\rho_{\sigma, \eta}(t)=e^{\sigma t}$. Let $\nu_{\sigma, \eta}=\gamma \mu_{\sigma, \eta}$, where $\gamma>0$. Then $\nu_{\sigma, \eta} \in \mathcal{M}$.

First, if for $|s| \leq r, r>0$, we write:

$$
J_{\sigma, \eta}(r, s):=\frac{1}{\rho_{\sigma, \eta}(s)} \int_{s}^{r}|k(t-s)| d \mu_{\sigma}(t),
$$

we must prove that:

$$
\sup _{|s| \leq r, r>0} J_{\sigma, \eta}(r, s)<\infty
$$

In fact,

$$
\begin{aligned}
J_{\sigma, \eta}(r, s) & =\frac{1}{10 e^{\sigma s}}\left(\int_{s}^{r} e^{-2(t-s)} e^{\sigma t} d t+\eta \sum_{s \leq n \leq r} e^{-2(n-s)} e^{\sigma n}\right) \\
& =\frac{1}{10}\left(\int_{s}^{r} e^{-(2-\sigma)(t-s)} d t+\eta \sum_{s \leq n \leq r} e^{-(2-\sigma)(n-s)}\right) \\
& \leq \frac{1}{10}\left(\int_{0}^{r-s} e^{-(2-\sigma) u} d u+\eta \sum_{[s] \leq n \leq[r]} e^{-(2-\sigma)(n-[s]-1)}\right),
\end{aligned}
$$

where we applied the change of integral $u=t-s$ in the integral and we denoted $[x]$ the integral part of the real number $x$. We next apply the change of index $m=n-[s]$ in the latter sum; this implies: 


$$
J_{\sigma, \eta}(r, s) \leq \frac{1}{10}\left(\int_{0}^{r-s} e^{-(2-\sigma)(u)} d u+\eta e^{2-\sigma} \sum_{m=0}^{[r]-[s]} e^{-(2-\sigma) m}\right)
$$

Then:

$$
\begin{aligned}
\sup _{|s| \leq r, r>0} J_{\sigma, \eta}(r, s) & \leq \frac{1}{10}\left(\int_{0}^{\infty} e^{-(2-\sigma)(u)} d u+\eta e^{2-\sigma} \sum_{m=0}^{\infty} e^{-(2-\sigma) m}\right) \\
& =\frac{1}{10(2-\sigma)}+\eta e^{2-\sigma} \frac{1}{10\left(1-e^{-(2-\sigma)}\right)}<\infty .
\end{aligned}
$$

This proves the estimate (3.1.1).

Secondly, we shall show that for all $\alpha>0$, we have:

$$
\lim _{r \rightarrow \infty} \frac{\mu_{\sigma, \eta}([-r, \alpha-r])}{\nu_{\sigma, \eta}([-r, r])}=0 .
$$

It actually suffices to prove this estimate when $\alpha$ is a positive integer. In fact,

$$
\begin{aligned}
\frac{\mu_{\sigma, \eta}([-r, \alpha-r])}{\nu_{\sigma, \eta}([-r, r])}= & \frac{\int_{-r}^{\alpha-r} e^{\sigma t} d t+\eta \sum_{-r \leq n \leq \alpha-r} e^{\sigma n}}{\gamma\left(\int_{-r}^{r} e^{\sigma t} d t+\eta \sum_{-r \leq n \leq r} e^{\sigma n}\right)} \\
\leq & \frac{\frac{1}{\sigma}\left(e^{\sigma(\alpha-r)}-e^{-\sigma r}\right)+\eta \sum_{-[r]-1 \leq n \leq \alpha-[r]} e^{\sigma n}}{\gamma\left(\frac{1}{\sigma}\left(e^{\sigma r}-e^{-\sigma r}\right)+\eta \sum_{-[r] \leq n \leq[r]} e^{\sigma n}\right)} \\
= & \frac{\frac{1}{\sigma} e^{-\sigma r}\left(e^{\sigma \alpha}-1\right)+\eta e^{-\sigma([r]+1)} \sum_{m=0}^{\alpha+1} e^{\sigma m}}{\left(\frac{1}{\sigma}\left(e^{\sigma r}-e^{-\sigma r}\right)+\eta e^{-\sigma[r]} \sum_{m=0}^{2[r]} e^{\sigma m}\right)}
\end{aligned}
$$

where we applied the change of index $m=n+[r]+1$ on the numerator and the change of index $m=n+[r]$ on the denominator. So

$$
\frac{\mu_{\sigma, \eta}([-r, \alpha-r])}{\mu_{\sigma, \eta}([-r, r])} \leq \frac{\frac{1}{\sigma} e^{-\sigma r}\left(e^{\sigma \alpha}-1\right)+\eta \frac{e^{\sigma(\alpha+2)}-1}{e^{\sigma}-1} e^{-\sigma([r]+1)}}{\frac{\gamma}{\sigma} e^{\sigma r}\left(1-e^{-2 \sigma r}\right)+\gamma \eta e^{-\sigma[r]} \frac{e^{\sigma(2[r]+1)}-1}{e^{\sigma}-1}} .
$$

The estimation (3.3.1) easily follows.

Thirdly, we show that $\left(\mathbf{H}_{1}\right)$ holds.

$$
\limsup _{r \rightarrow \infty} \frac{\mu_{\sigma, \eta}([-r, r])}{\nu_{\sigma, \eta}([-r, r])}=\frac{1}{\gamma}<\infty .
$$

Then, Theorem 3.1 and Corollary 3.5 hold. 


\subsection{Translation invariance and convolution invariance of $P A P(\mathbb{R}, X, \mu, \nu)$ and $P A A(\mathbb{R}, X, \mu, \nu)$}

Theorem 3.7. Assume that $\mu, \nu \in \mathcal{M}$ and $\left(\boldsymbol{H}_{1}\right)$ holds. If the space $\mathcal{E}(\mathbb{R}, X, \mu, \nu)$ is translation invariant, then $\mathcal{E}(\mathbb{R}, X, \mu, \nu)$ is convolution invariant.

Proof. Let $f \in \mathcal{E}(\mathbb{R}, X, \mu, \nu)$. Let us prove that if $f(t-\tau) \in \mathcal{E}(\mathbb{R}, X, \mu, \nu)$, for $\tau \in \mathbb{R}$, then $\zeta f \in \mathcal{E}(\mathbb{R}, X, \mu, \nu)$, i.e. $\mathbf{M}(\zeta f, \mu, \nu)=0$. By the properties of convolution we have that $f \in B C(\mathbb{R}, X)$ implies that $k * f \in B C(\mathbb{R}, X), \forall k \in L^{1}(\mathbb{R})$. By the Fubini's Theorem we have,

$$
\begin{aligned}
\mathbf{M}(\zeta f, \mu, \nu) & =\lim _{r \rightarrow+\infty} \frac{1}{\nu([-r, r])} \int_{-r}^{r}\|k * f\|_{X} d \mu(t) \\
& \leq \lim _{r \rightarrow+\infty} \int_{-\infty}^{\infty} \frac{|k(s)|}{\nu([-r, r])}\left(\int_{-r}^{r}\|f(t-s)\|_{X} d \mu(t)\right) d s
\end{aligned}
$$

Since $f$ is invariant by translation we have for all $s \in \mathbb{R}$ :

$$
\lim _{r \rightarrow+\infty} \frac{1}{\nu([-r, r])} \int_{-r}^{r}\|f(t-s)\|_{X} d \mu(t)=0
$$

Since $\left(\mathbf{H}_{1}\right)$ holds for all $s \in \mathbb{R}$, we have that:

$$
0 \leq \frac{|k(s)|}{\nu([-r, r])} \int_{-r}^{r}\|f(t-s)\|_{X} d \mu(t) d s \leq \beta|k(s)|\|f\|_{\infty},
$$

where $k \in L^{1}(\mathbb{R})$. Then by the Lebesgue dominated convergence Theorem, we obtain that $\mathbf{M}(\zeta f, \mu, \nu)=0$.

Theorem 3.8. Let $\left(\boldsymbol{H}_{1}\right)$ holds. If the space $P A P(\mathbb{R}, X, \mu, \nu)$ (resp. $\left.P A A(\mathbb{R}, X, \mu, \nu)\right)$ is translation invariant, then $\mathcal{E}(\mathbb{R}, X, \mu, \nu)$ is convolution invariant.

Proof. For $f \in A P(\mathbb{R}, X)$ or $f \in A A(\mathbb{R}, X)$, then $f$ is invariant by $\zeta$ i.e. $\zeta f \in A P(\mathbb{R}, X)$ or $\zeta f \in A A(\mathbb{R}, X)$. We use the previous theorem to conclude.

Corollary 3.9. Let $\left(\boldsymbol{H}_{0}\right)$ and $\left(\boldsymbol{H}_{1}\right)$ hold. Then $\mathcal{E}(\mathbb{R}, X, \mu, \nu), P A P(\mathbb{R}, X, \mu, \nu)$ and $P A A(\mathbb{R}, X, \mu, \nu)$ are convolution invariant.

Proof. Combine Theorem 2.12 and Theorem 3.8. 


\section{Existence, Uniqueness results and Applications}

This section is similar to section 3 in [11], but here we applied our new results obtained in the above section.

\subsection{Evolution Families and Exponential Dichotomy}

$\left(\mathbf{H}_{2}\right)$ : A family of closed linear operators $A(t)$ for $t \in \mathbb{R}$ on $X$ with domain $D(A(t)$ ) (possibly not densely defined), is said to satisfy the so-called Acquistapace-Terreni conditions, if there exist constants $\omega \in \mathbb{R}, \theta \in\left(\frac{\pi}{2}, \pi\right), K, L \geq 0$ and $\mu_{0}, \nu_{0} \in(0,1]$, with $1<\mu_{0}+\nu_{0}$ such that

$$
\Sigma_{\theta} \cup\{0\} \subset \rho(A(t)-\omega) \ni \lambda,\|R(\lambda, A(t)-\omega)\| \leq \frac{K}{1+|\lambda|},
$$

and

$$
\|(A(t)-\omega) R(\lambda, A(t)-\omega)[R(\omega, A(t))-R(\omega, A(s))]\| \leq L \frac{|t-s|^{\mu_{0}}}{|\lambda|^{\nu_{0}}},
$$

for $t, s \in \mathbb{R}, \lambda \in \Sigma_{\theta}:=\{\lambda \in \mathbb{C} /\{0\}:|\arg \lambda| \leq \theta\}$.

For a given family of linear operators $A(t)$, the existence of an evolution family associated with it is not always guaranteed. However, if $A(t)$ satisfied Acquistapace-Terreni conditions, then there exists a unique evolution family

$$
\mathcal{U}=\{\mathcal{U}(t, s): t, s \in \mathbb{R}, t \geq s\}
$$

on $X$ associated with $A(t)$ such that $\mathcal{U}(t, s) X \subseteq D(A(t))$ for all $t, s \in \mathbb{R}$ with $t \geq s$, and,

i) $\mathcal{U}(t, r) \mathcal{U}(r, s)=\mathcal{U}(t, s)$ and $\mathcal{U}(s, s)=I \forall t \geq r \geq s$ and $t, r, s \in \mathbb{R}$;

ii) the map $(t, s) \longrightarrow \mathcal{U}(t, s) x$ is continuous for all $x \in X, t \geq s$ and $t, s \in \mathbb{R}$;

iii) $\mathcal{U}(., s) \in C^{1}((s, \infty), B(X)), \frac{\partial \mathcal{U}}{\partial t}(t, s)=A(t) \mathcal{U}(t, s)$ and

$$
\left\|A(t)^{k} \mathcal{U}(t, s)\right\| \leq K(t-s)^{-k}
$$

for $0<t-s \leq 1, k=0,1$.

Definition $4.1([3])$. An evolution family $(\mathcal{U}(t, s))_{t \geq s}$ on a Banach space $X$ is called hyperbolic (or has exponential dichotomy) if there exist projections $P(t), t \in \mathbb{R}$, uniformly bounded and strongly continuous in $t$, and constants $N \geq 1, \delta>0$ such that

i) $\mathcal{U}(t, s) P(s)=P(t) \mathcal{U}(t, s)$ for $t \geq s$;

ii) the restriction $\mathcal{U}_{Q}(t, s): Q(s) X \longrightarrow Q(t) X$ for $\mathcal{U}(t, s)$ is inversible for $t, s \in \mathbb{R}$ and we set

$$
\mathcal{U}_{Q}(t, s)=\mathcal{U}(s, t)^{-1}
$$


iii)

$$
\|\mathcal{U}(t, s) P(s)\| \leq N e^{-\delta(t-s)}
$$

and

$$
\left\|\mathcal{U}_{Q}(s, t) Q(t)\right\| \leq N e^{-\delta(t-s)}
$$

for $t \geq s$ and $t, s \in \mathbb{R}$, where $Q(t):=I-P(t)$

\subsection{Existence Results}

To study the existence and uniqueness of $(\mu, \nu)$ - pseudo-almost periodic (respectively, $(\mu, \nu)$ pseudo-almost automorphic) solutions to equation (1.1), we also assume that the next hypothesis holds:

$\left(\mathbf{H}_{3}\right)$ The evolution family $\mathbb{U}$ generated by $A($.$) has an exponential dichotomy with constants$ $N \geq 1, \delta>0$ and dichotomy projections $P(t)$.

We recall the following sufficient conditions to fulfill the assumption $\left(\mathbf{H}_{3}\right)$.

$\left(\mathbf{H}_{3.1}\right) \quad$ Let $(A(t), D(A(t)))_{t \in \mathbb{R}}$ be generators of analytic semigroups on $X$ of the same type. Suppose that $D(A(t))=D(A(0)), A(t)$ is inversible, $\sup _{t, s \in \mathbb{R}}\left\|A(t) A(s)^{-1}\right\|$ is finite, and

$$
\left\|A(t) A(s)^{-1}-I\right\| \leq L_{0}|t-s|^{\mu_{1}}
$$

for $t, s \in \mathbb{R}$ and constants $L_{0} \geq 0$ and $0 \leq \mu_{1} \leq 1$.

$\left(\mathbf{H}_{3.2}\right)$ The semigroup $\left(e^{\tau A(t)}\right)_{\tau \geq 0}, t \in \mathbb{R}$, are hyperbolic with projection $P_{t}$ and constants $N, \delta>$ 0 . Moreover, let

$$
\left\|A(t)\left(e^{\tau A(t)} P_{t}\right)\right\| \leq \Psi(\tau), \quad\left\|A(t)\left(e^{\tau A(t)} Q_{t}\right)\right\| \leq \Psi(-\tau)
$$

for $\tau>0$ and a function $\Psi$ such that $\mathbb{R} \ni s \longrightarrow \varphi(s):=|s|^{\mu} \Psi(s)$ is integrable with $L_{0}\|\varphi\|_{L^{1}(\mathbb{R})}<1$.

We introduce here the defnition of the mild solution of equation (1.1).

Definition $4.2([3])$. A continuous function $u: \mathbb{R} \longmapsto X$ is called a bounded mild solution of equation (1.1) if:

$$
u(t)=\mathcal{U}(t, s) u(s)+\int_{s}^{t} \mathcal{U}(t, \tau) F(\tau, u(\tau)) d \tau, \forall t, s \in \mathbb{R}, \text { with } \quad t \geq s .
$$

Theorem $4.3([11])$. Assume that $\left(\boldsymbol{H}_{2}\right)$ and $\left(\boldsymbol{H}_{3}\right)$ hold. If there exists $0<K_{F}<\frac{\delta}{2 N}$ such that

$$
\|F(t, u)-F(t, v)\| \leq K_{F}\|u-v\|
$$

for all $u, v \in X$ and $t \in \mathbb{R}$, then the equation (1.1) has a unique bounded mild solution $u: \mathbb{R} \longmapsto X$ given by

$$
u(t)=\int_{\mathbb{R}} \Gamma(t, s) F(s, u(s)) d s, \quad t \in \mathbb{R},
$$


where the operator family $\Gamma(t, s)$, called Green's function corresponding to $\mathcal{U}$ and $P(\cdot)$, is given by

$$
\begin{gathered}
\Gamma(t, s)=\mathcal{U}(t, s) P(s), \quad \forall t, s \in \mathbb{R}, \text { with } \quad t \geq s, \\
\Gamma(t, s)=-\mathcal{U}_{Q}(t, s) Q(s), \quad \forall t, s \in \mathbb{R}, \text { with } \quad t<s .
\end{gathered}
$$

Denote by $\Gamma_{1}$ and $\Gamma_{2}$ the nonlinear integral operators defined by,

$$
\left(\Gamma_{1} u\right)(t):=\int_{-\infty}^{t} \mathcal{U}(t, s) P(s) F(s, u(s)) d s
$$

and

$$
\left(\Gamma_{2} u\right)(t):=\int_{t}^{+\infty} \mathcal{U}_{Q}(t, s) Q(s) F(s, u(s)) d s .
$$

In the rest of this work, we fix $\mu, \nu \in \mathcal{M}$ to satisfy $\left(\mathbf{H}_{1}\right)$.

\subsection{Existence of $(\mu, \nu)$-pseudo-almost periodic solutions}

In addition to the previous assumptions, we require the following additional ones:

$\left(\mathbf{H}_{4}\right): R(\omega, A().) \in A P(\mathbb{R}, \mathcal{L}(X))$.

$\left(\mathbf{H}_{5}\right)$ : We propose $F: \mathbb{R} \times X \longmapsto X$ belongs to $P A P(\mathbb{R} \times X, X, \mu, \nu)$ and there exists $K_{F}>0$ such that

$$
\|F(t, u)-F(t, v)\| \leq K_{F}\|u-v\|
$$

for all $u, v \in X$ and $t \in \mathbb{R}$.

The following Lemma plays an important role to prove the main results of this study.

Lemma $4.4([13])$. Assume that $\left(\boldsymbol{H}_{2}\right)-\left(\boldsymbol{H}_{4}\right)$ hold. Then $r \longrightarrow \Gamma(t+r, s+r)$ belongs to $A P(\mathbb{R}, \mathcal{L}(X))$ for all $t, s \in \mathbb{R}$, where we may take the same pseudo periods for $t, s$ with $|t-s| \geq h>0$. If $f \in A P(\mathbb{R}, \mathcal{L}(X))$, then the unique bounded mild solution $u(t)=\int_{\mathbb{R}} \Gamma(t, s) f(s) d s$ of the following equation

$$
u^{\prime}(t)=A(t) u(t)+f(t), \quad t \in \mathbb{R},
$$

is almost periodic.

Lemma 4.5. Assume that $\left(\boldsymbol{H}_{2}\right)-\left(\boldsymbol{H}_{5}\right)$ hold. If (3.1) and (3.2), or (3.1) and (3.3) hold, then the integral operators $\Gamma_{1}$ and $\Gamma_{2}$ defined above map $P A P(\mathbb{R}, X, \mu, \nu)$ into itself.

Proof. Let $u \in P A P(\mathbb{R}, X, \mu, \nu)$. setting $h(t)=F(t, u(t))$, using the assumption $\left(\mathbf{H}_{5}\right)$ and Theorem 2.16 it follows that $h \in P A P(\mathbb{R}, X, \mu, \nu)$. Now write $h=\Psi_{1}+\Psi_{2}$ where $\Psi_{1} \in A P(\mathbb{R}, X)$ and $\Psi_{2} \in \mathcal{E}(\mathbb{R}, X, \mu, \nu)$. That is, $\Gamma_{1} h=\Xi\left(\Psi_{1}\right)+\Xi\left(\Psi_{2}\right)$ where

$$
\Xi \Psi_{i}(t):=\int_{-\infty}^{t} U(t, s) P(s) \Psi_{i}(s) d s, \text { for } \quad i \in\{1,2\} .
$$


From Lemma 4.4, we have $\Xi\left(\Psi_{1}\right) \in A P(\mathbb{R}, X)$. To complete the proof, we will prove that $\Xi\left(\Psi_{2}\right) \in$ $\mathcal{E}(\mathbb{R}, X, \mu, \nu)$. Now, let $r>0$. From equation (4.3), we have:

$$
\begin{aligned}
\frac{1}{\nu([r,-r])} \int_{-r}^{r} \|\left(\Xi\left(\Psi_{2}\right)(t) \| d \mu(t)\right. & \leq \frac{1}{\nu([r,-r])} \int_{-r}^{r} \int_{-\infty}^{t} U(t, s) P(s) \Psi_{2}(s) d s d \mu(t) \\
& \leq \frac{N}{\nu([r,-r])} \int_{-r}^{r} \int_{-\infty}^{t} e^{-\delta(t-s)}\left\|\Psi_{2}(s)\right\| d s d \mu(t)
\end{aligned}
$$

Since $\mu$ and $\nu$ satisfy (3.1.1) and (3.2.1), (3.1.1) and (3.3.1), with $k(t)=e^{-\delta t}$, then by Theorem 3.1 or Corollary 3.5 , we conclude that:

$$
\lim _{r \rightarrow+\infty} \frac{1}{\nu([r,-r])} \int_{-r}^{r} \|\left(\Xi\left(\Psi_{2}\right)(t) \| d \mu(t)=0 .\right.
$$

The proof for $\Gamma_{2} u($.$) is similar to that of \Gamma_{1} u($.$) except that one makes use of equation (4.4) instead$ of (4.3), (3.1.2) and (3.2.2), or (3.1.2) and (3.3.2).

Theorem 4.6. Assume that $\left(\boldsymbol{H}_{2}\right)-\left(\boldsymbol{H}_{5}\right)$ hold. If (3.1) and (3.2), or (3.1) and (3.3) hold, then equation (1.1) has a unique $(\mu, \nu)$-pseudo almost periodic mild solution whenever $K_{F}$ is small enough.

Proof. Consider the nonlinear operator $\mathbb{K}$ defined on $P A P(\mathbb{R}, X, \mu, \nu)$ by

$$
\mathbb{K} u(t)=\int_{-\infty}^{t} U(t, s) P(s) F(s, u(s)) d s-\int_{t}^{+\infty} U_{Q}(t, s) Q(s) F(s, u(s)) d s, \quad \forall t \in \mathbb{R} .
$$

By Lemma 4.5, it follows that $\mathbb{K}$ maps $P A P(\mathbb{R}, X, \mu, \nu)$ into itself. To complete the proof one has to show that $\mathbb{K}$ is a contraction map on $P A P(\mathbb{R}, X, \mu, \nu)$.

Let $u, v \in P A P(\mathbb{R}, X, \mu, \nu)$. Firstly, we have that:

$$
\begin{aligned}
\left\|\Gamma_{1}(v)(t)-\Gamma_{1}(u)(t)\right\| & \leq \int_{-\infty}^{t}\|U(t, s) P(s)[F(s, v(s))-F(s, u(s))]\| d s \\
& \leq N K_{F} \int_{-\infty}^{t} e^{-\delta(t-s)}\|v(s)-u(s)\| d s \\
& \leq N K_{F} \delta^{-1}\|v-u\|_{\infty} .
\end{aligned}
$$

Next, we have that:

$$
\begin{aligned}
\left\|\Gamma_{2}(v)(t)-\Gamma_{2}(u)(t)\right\| & \leq \int_{t}^{+\infty}\left\|U_{Q}(t, s) Q(s)[F(s, v(s))-F(s, u(s))]\right\| d s \\
& \leq N K_{F} \int_{t}^{+\infty} e^{-\delta(t-s)}\|v(s)-u(s)\| d s \\
& \leq N K_{F} \delta^{-1}\|v-u\|_{\infty} \int_{t}^{+\infty} e^{-\delta(t-s)} d s \\
& =N K_{F} \delta^{-1}\|v-u\|_{\infty} .
\end{aligned}
$$

Finally, combining previous approximations it follows that:

$$
\|\mathbb{K} v-\mathbb{K} u\|_{\infty}<2 N K_{F} \delta^{-1}\|v-u\|_{\infty} .
$$


Thus if $K_{F}$ is small enough, that is, $K_{F}<\delta(2 N)^{-1}$, then $\mathbb{K}$ is a contraction map on $P A P(\mathbb{R}, X, \mu, \nu)$. Therefore, $\mathbb{K}$ has a unique fixed point in $P A P(\mathbb{R}, X, \mu, \nu)$, that is, there exists a unique function $u$ satisfying $\mathbb{K} u=u$, which is the unique $(\mu, \nu)$-pseudo almost periodic mild solution to equation (1.1).

Theorem $4.7([11])$. Assume that $\left(\boldsymbol{H}_{2}\right)-\left(\boldsymbol{H}_{5}\right)$ hold. If $\left(\boldsymbol{H}_{0}\right)$ holds, then equation (1.1) has a unique $(\mu, \nu)$-pseudo almost periodic mild solution whenever $K_{F}$ is small enough.

\subsection{Existence of $(\mu, \nu)$-pseudo-almost automorphic solutions}

In this section we consider the following assumptions:

$\left(\mathbf{H}_{6}\right): R(\omega, A().) \in A A(\mathbb{R}, \mathcal{L}(X))$.

$\left(\mathbf{H}_{7}\right)$ : We propose $F: \mathbb{R} \times X \longmapsto X$ belongs to $P A A(\mathbb{R} \times X, X, \mu, \nu)$ and there exists $K_{F}>0$ such that

$$
\|F(t, u)-F(t, v)\| \leq K_{F}\|u-v\|_{\infty}
$$

for all $u, v \in X$ and $t \in \mathbb{R}$.

Lemma $4.8([14])$. Assume that $\left(\boldsymbol{H}_{2}\right),\left(\boldsymbol{H}_{3}\right)$ and $\left(\boldsymbol{H}_{6}\right)$ hold. Let a sequence $\left(s_{l}^{\prime}\right)_{l \in \mathbb{N}} \subset \mathbb{R}$ there is a sub-sequence $\left(s_{l}\right)_{l \in \mathbb{N}}$ such that for every $h>0$

$$
\left\|\Gamma\left(t+s_{l}-s_{k}, s+s_{l}-s_{k}\right)-\Gamma(t, s)\right\| \longrightarrow 0, \quad k, l \longrightarrow \infty .
$$

Lemma 4.9. Assume that $\left(\boldsymbol{H}_{2}\right),\left(\boldsymbol{H}_{3}\right),\left(\boldsymbol{H}_{6}\right)$ and $\left(\boldsymbol{H}_{7}\right)$ hold. If (3.1) and (3.2) or (3.1) and (3.3) or $\left(\boldsymbol{H}_{0}\right)$ hold, then the integral operators $\Gamma_{1}$ and $\Gamma_{2}$ defined above map $P A A(\mathbb{R} \times X, X, \mu, \nu)$ into itself.

Proof. Let $u \in P A A(\mathbb{R}, X, \mu, \nu)$. Setting $g(t)=F(t, u(t))$, by assumption $\left(\mathbf{H}_{7}\right)$ and Theorem 2.17 we obtain that $g \in P A A(\mathbb{R}, X, \mu, \nu)$. Now write $g=u_{1}+u_{2}$ where $u_{1} \in A A(\mathbb{R}, X)$ and $u_{2} \in \mathcal{E}(\mathbb{R}, X, \mu, \nu)$. That is, $\Gamma_{1} g=\mathcal{S} u_{1}+\mathcal{S} u_{2}$, where

$$
\mathcal{S} u_{1}(t):=\int_{-\infty}^{t} U(t, s) P(s) u_{1}(s) d s, \quad \mathcal{S} u_{2}(t):=\int_{-\infty}^{t} U(t, s) P(s) u_{2}(s) d s .
$$

From equation (4.3), we obtain:

$$
\left\|\mathcal{S} u_{1}(t)\right\| \leq N \delta^{-1}\left\|u_{1}\right\|_{\infty}, \quad\left\|\mathcal{S} u_{2}(t)\right\| \leq N \delta^{-1}\left\|u_{2}\right\|_{\infty}, \quad \forall t \in \mathbb{R} .
$$

Then $\mathcal{S} u_{1}(t), \mathcal{S} u_{2}(t) \in B C(\mathbb{R}, X)$. Now, we prove that $\mathcal{S} u_{1}(t) \in A A(\mathbb{R}, X)$. Since $u_{1} \in A A(\mathbb{R}, X)$, then for every sequence $\left(\tau_{n}^{\prime}\right)_{n \in \mathbb{N}} \in \mathbb{R}$ there exists a subsequence $\left(\tau_{n}\right)_{n \in \mathbb{N}}$ such that:

$$
v_{1}(t):=\lim _{n \rightarrow \infty} u_{1}\left(t+\tau_{n}\right)
$$

is well defined for each $t \in \mathbb{R}$, and

$$
\lim _{n \rightarrow \infty} v_{1}\left(t-\tau_{n}\right)=u_{1}(t), \forall t \in \mathbb{R} .
$$


Set for $t \in \mathbb{R}$,

$$
M(t):=\int_{-\infty}^{t} U(t, s) P(s) u_{1}(s) d s, \text { and } N(t):=\int_{-\infty}^{t} U(t, s) P(s) v_{1}(s) d s .
$$

Now, we have

$$
\begin{aligned}
M\left(t+\tau_{n}\right)-N(t) & =\int_{-\infty}^{t+\tau_{n}} U\left(t+\tau_{n}, s\right) P(s) u_{1}(s) d s-\int_{-\infty}^{t} U(t, s) P(s) v_{1}(s) d s \\
& =\int_{-\infty}^{t} U\left(t+\tau_{n}, s+\tau_{n}\right) P\left(s+\tau_{n}\right) u_{1}\left(s+\tau_{n}\right) d s \\
& -\int_{-\infty}^{t} U(t, s) P(s) v_{1}(s) d s \\
& =\int_{-\infty}^{t} U\left(t+\tau_{n}, s+\tau_{n}\right) P\left(s+\tau_{n}\right)\left[u_{1}\left(s+\tau_{n}\right)-v_{1}(s)\right] d s \\
& +\int_{-\infty}^{t}\left[U\left(t+\tau_{n}, s+\tau_{n}\right) P\left(s+\tau_{n}\right)-U(t, s) P(s)\right] v_{1}(s) d s .
\end{aligned}
$$

Using equation (4.3), equation (4.6) and the Lebesgue's Dominated Convergence Theorem, it follows that:

$$
\lim _{n \rightarrow+\infty}\left\|\int_{-\infty}^{t} U\left(t+\tau_{n}, s+\tau_{n}\right) P\left(s+\tau_{n}\right)\left[u_{1}\left(s+\tau_{n}\right)-v_{1}(s)\right] d s\right\|=0, \text { for } t \in \mathbb{R} .
$$

Similary, using Lemma 4.8 it follows that:

$$
\lim _{n \rightarrow+\infty}\left\|\int_{-\infty}^{t}\left[U\left(t+\tau_{n}, s+\tau_{n}\right) P\left(s+\tau_{n}\right)-U(t, s) P(s)\right] v_{1}(s) d s\right\|=0, \text { for } \quad t \in \mathbb{R} .
$$

Therefore, we have that:

$$
N(t):=\lim _{n \rightarrow \infty} M\left(t+\tau_{n}\right), \forall t \in \mathbb{R} .
$$

Using similar ideas as the previous ones, then:

$$
M(t):=\lim _{n \rightarrow \infty} N\left(t-\tau_{n}\right), \forall t \in \mathbb{R} .
$$

Therefore, $\mathcal{S} u_{1}(t) \in A A(\mathbb{R}, X)$. Arguing as in Lemma 4.5, we get that $\mathcal{S} u_{2}(t) \in \mathcal{E}(\mathbb{R}, X, \mu, \nu)$. The proof for $\Gamma_{2} u($.$) is similar to that of \Gamma_{1} u($.$) except that one makes use of equation (4.4) instead of$ equation (4.3) and, (3.1.2) and (3.2.2), (3.1.2) and (3.3.2).

Theorem 4.10. Under assumptions $\left(\boldsymbol{H}_{2}\right),\left(\boldsymbol{H}_{3}\right),\left(\boldsymbol{H}_{6}\right)$ and $\left(\boldsymbol{H}_{7}\right)$, if (3.1) and (3.2) or (3.1) and $(3.3)$ or $\left(\boldsymbol{H}_{0}\right)$ then equation $(1.1)$ has a unique $(\mu, \nu)$-pseudo almost automorphic mild solution whenever $K_{F}$ is small enough.

Proof The proof of Theorem 4.10 is similar to that Theorem 4.6 except that one makes use of Lemma 4.9 instead of Lemma 4.5. 


\subsection{Neutral Systems}

In this subsection, we establish the existence and uniqueness of $(\mu, \nu)$-pseudo almost periodic (respectively $(\mu, \nu)$-pseudo almost automorphic) solutions for the nonautonomous neutral partial evolution equation (1.2). For that, we need the following assumptions:

$\left(\mathbf{H}_{8}\right)$ : We suppose $G: \mathbb{R} \times X \longrightarrow X$ belongs to $P A P(\mathbb{R} \times X, X, \mu, \nu)$ and there exists $K_{G}>0$ such that:

$$
\|G(t, u)-G(t, v)\| \leq K_{G}\|u-v\|
$$

for all $u, v \in X$ and $t \in \mathbb{R}$.

$\left(\mathbf{H}_{9}\right)$ We suppose $G: \mathbb{R} \times X \longrightarrow X$ belongs to $P A A(\mathbb{R} \times X, X, \mu, \nu)$ and there exists $K_{G}>0$ such that:

$$
\|G(t, u)-G(t, v)\| \leq K_{G}\|u-v\|
$$

for all $u, v \in X$ and $t \in \mathbb{R}$.

Definition 4.11. A function $v: \mathbb{R} \longmapsto X$ is said a mild solution of $(1.2)$ on $\mathbb{R}$ if :

$$
v(t)=G(t, v(t))+\int_{-\infty}^{t} U(t, s) P(s) F(s, v(s)) d s-\int_{t}^{+\infty} U_{Q}(t, s) Q(s) F(s, v(s)) d s,
$$

for all $t \in \mathbb{R}$.

Theorem 4.12. Assume that assumptions $\left(\boldsymbol{H}_{2}\right)-\left(\boldsymbol{H}_{5}\right)$ and $\left(\boldsymbol{H}_{8}\right)$ hold. If (3.1) and (3.2) or (3.1) and (3.3) or $\left(\boldsymbol{H}_{0}\right)$ hold, and $\left(K_{G}+2 N K_{F} \delta^{-1}\right)<1$, then equation (1.2) has a unique $(\mu, \nu)$-pseudo almost periodic mild solution.

Proof. We consider the nonlinear operator $\mathbb{W}$ defined on $P A P(\mathbb{R}, X, \mu, \nu)$ by:

$$
\mathbb{W} v(t)=G(t, v(t))+\int_{-\infty}^{t} U(t, s) P(s) F(s, v(s)) d s-\int_{t}^{+\infty} U_{Q}(t, s) Q(s) F(s, v(s)) d s
$$

for all $t \in \mathbb{R}$. From $\left(\mathbf{H}_{9}\right)$, Theorem 2.16, and Lemma 4.5 it follows that $\mathbb{W}$ maps $P A P(\mathbb{R}, X, \mu, \nu)$ into itself. To complete the proof we need to show that $\mathbb{W}$ is a contraction map on $P A P(\mathbb{R}, X, \mu, \nu)$. For that, letting $u, v \in P A P(\mathbb{R}, X, \mu, \nu)$, we obtain:

$$
\|\mathbb{W} v-\mathbb{W} u\|_{\infty} \leq\left(K_{G}+2 N K_{F} \delta^{-1}\right)\|v-u\|_{\infty},
$$

which yields $\mathbb{W}$ is a contraction map on $P A P(\mathbb{R}, X, \mu, \nu)$. Therefore, $\mathbb{W}$ has unique fixed point in $P A P(\mathbb{R}, X, \mu, \nu)$. Therefore, equation (1.2) has unique $(\mu, \nu)$-pseudo almost periodic mild solution.

Theorem 4.13. Assume that $\left(\boldsymbol{H}_{2}\right),\left(\boldsymbol{H}_{3}\right),\left(\boldsymbol{H}_{6}\right),\left(\boldsymbol{H}_{7}\right)$ and $\left(\boldsymbol{H}_{9}\right)$ hold and $\left(K_{G}+2 N K_{F} \delta^{-1}\right)<1$. If (3.1) and (3.2) or (3.1) and (3.3) or $\left(\boldsymbol{H}_{0}\right)$ hold, then equation (1.2) has a unique $(\mu, \nu)$-pseudo almost automorphic mild solution.

Proof. Similarly, we can show, by using the assumption $\left(\mathbf{H}_{9}\right)$, Theorem 2.17 and Lemma 4.9, that the equation (1.2) has a unique $(\mu, \nu)$-pseudo almost automorphic mild solution. 


\section{Acknowledgments}

The authors are grateful to the anonymous referee for the careful reading of this paper, for very helpful suggestions and comments which improved of quality of this article.

\section{References}

[1] P. Acquistapace, F. Flandoli, and B. Terreni, "Initial boundary value problems and optimal control for nonautonomous parabolic systems", SIAM Journal on Control and Optimization, vol. 29, pp. 89-118, 1991.

[2] P. Acquistapace, and B. Terreni, "A unified approach to abstract linear nonautonomous parabolic equations", Rendiconti del Seminario Matematico della Università di Padova, vol. 78, pp. 47-107, 1987.

[3] M. Baroun, S. Boulite, G. M. N'Guérékata, and L. Maniar, "Almost automorphy of semilinear parabolic evolution equations", Electronic Journal of Differential Equations, vol. 60, pp. 1-9, 2008 .

[4] J. Blot, P. Cieutat, and K. Ezzinbi, "Measure theory and almost automorphic functions: new developments and applications", Nonlinear Analysis, vol. 75, pp. 2426-2447, 2012.

[5] J. Blot, P. Cieutat, and K. Ezzinbi, "New approach for weighted pseudo almost periodic functions under the light of measure theory, basic results and applications", Applicable Analysis, vol. 92, no. 3, pp. 493-526, 2013.

[6] C. Corduneanu, Almost Periodic Functions, Wiley, New York, 1968, (Reprinted, Chelsea, New York, 1989).

[7] A. Coronel, M. Pinto, and D. Sepulveda, "Weighted pseudo almost periodic functions, convolutions and abstract integral equations", J. Math. Anal. Appl., vol. 435, pp. 1382-1399, 2016.

[8] T. Diagana, "Double weighted pseudo-almost periodic functions", Afr. Diaspora J. Math., vol. 12, pp. 121-136, 2011.

[9] T. Diagana, "Existence of weighted pseudo almost periodic solutions to some classes of nonautonomous partial evolution equations", Nonlinear Analysis, vol. 74, pp. 600-615, 2011.

[10] T. Diagana, "Pseudo-almost periodic solutions to some classes of nonautonomous partial evolution equations", Journal of the Franklin Institute, vol. 348, pp. 2082-2098, 2011. 
[11] T. Diagana, K. Ezzinbi, and M. Miraoui, "Pseudo-Almost Periodic and Pseudo-Almost Automorphic solutions to Some Evolution Equations Involving Theorical Measure Theory", CUBO A Mathematical Journal, vol. 16, no. 2, pp. 01-31, 2014.

[12] M. Fréchet, "Sur le théorème ergodique de Birkhoff", Les comptes Rendus Mathématiques de l'Académie de Sciences Paris, vol. 213, pp. 607-609, 1941.

[13] A. Haraux, Systèmes dynamiques et dissipatifs et applications, Recherches en Mathématiques Appliquées, Masson, Paris, 1991.

[14] L. Maniar, and R. Schnaubelt, "Almost periodicity of inhomogeneous parabolic evolution equations", Lecture Notes in Pure and Applied Mathematics, vol. 234, pp. 299-318, 2003.

[15] F. Mbounja Béssémè, D. Békollè, K. Ezzinbi, S. Fatajou, and D.E. Houpa Danga, "Convolution in $\mu$-pseudo almost periodic and $\mu$-pseudo almost automorphic functions spaces and applications to solve Integral equations", Nonautonomous Dynamical Systems, vol. 7, pp. $32-52,2020$.

[16] G. M. N'Guérékata, Topics in Almost automorphy, Springer, New York, Boston, London, Moscow, 2005.

[17] H. L. Royden, Real Analysis, Third edition, Macmillan Publishing Company, New York, 1988. 